\title{
Routing Algorithm of Concentric Excursions for ITS Communication
}

\author{
Jianfeng Luo ${ }^{1}$, Yuqiang Chen ${ }^{1}$ \\ ${ }^{1}$ Department of Computer Engineering, Dongguan Polytechnic, Dongguan Guangdong 523808, China
}

\begin{abstract}
In the modern society, intelligent transportation builds on the basic of real-time wireless communication with vast information. Only by adding and upgrading the high-costed equipment to keep the speed of communication with the development of the transportation is far from enough ${ }^{[1]}$. Thus, to make full use of the present equipment to achieve higher speed of information transmission is a practical and challenging project. The Routing Algorithm of this paper is taking the Traffic Management Center as the center. Starting from the center and covering all the traffic management station, we can get the best path program under multi-limits with the help of the combination Algorithm of Shortest Path Algorithms and Concentric Excursions. A lot of constraints has been taken into consideration so as to make the result preciser. The result of multiple experiments has testified the feasibility and efficiency of the algorithm even under the great information content.
\end{abstract}

Key words: Cumulative Conditions Queue, multi-limits Routing Algorithm, Concentric Excursions, value-Saving Transmission Queue, Shortest Path

\section{Introduction}

Traffic monitoring information grows with the rapid growth of the cars number. To do researches on the routing algorithm of information transmission to improve the speed is of great importance. ${ }^{[2]}$ The present common wireless networks are Cellular System with complicated structure and Network Protocol which may cause problems such as louder noise, longer Transmission time, worse net jam etc. ${ }^{[3]}$ The wide range of intelligent traffic management, a large amount of equipment, complicated routing paths and huge amount of information will force the system to upgrade continuously. Otherwise, the system has to keeping adding new equipment or other high-costed way to keep up with the need of the pace ${ }^{[4]}$. In order to get rid of the problems mentioned above, we have to figure out a simple-structured and time-saving routing solution which can cover all the connecting points as well work under the limitation of transmission path and data.

\section{A Brief introduction of the intelligent transportation wireless network}

Shown in Fig.1, the Traffic Management Center is responsible for the calculations of traffic control and coordination. The result will be sent back to the regional wireless receiving tower through wireless signal routing. The receiving tower with the relay function will send the information to the assigned server and then to the roadside equipment or to the mobile equipment fixed in the vehicle. Meanwhile, the traffic signal will be sent through the reverse way back to the Traffic Management Center ${ }^{[5]}$. 


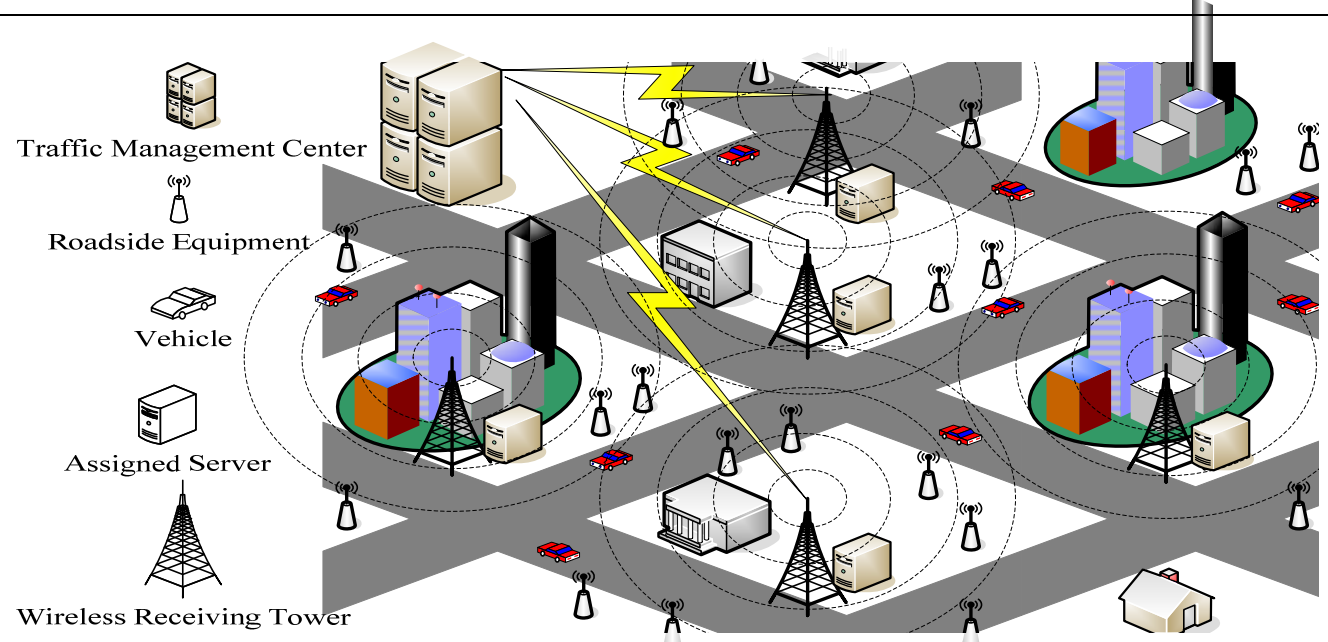

Fig.1 the Wireless Network Model for the Intelligent Transportation System

\section{The Core idea of Excursion Algorithm}

The Core idea of Excursion Algorithm is as follow: signal from Traffic Management Center covers as many as the receiving towers and collects as much as the local traffic information at the same time. What works importantly is to work out the shortest path to send the information back to the Traffic Management Center. Suppose that the information is fairly large and take up all the broadband resource, we can only exchange the information in the next tower. Under such situation, we have to prove that the Excursion Algorithm is the shortest Information transmission path among the receiving towers.

The following simple Model Diagram will show the wireless traffic network $^{[6]}$ (Fig.2): $\mathrm{C}$ is the Traffic Management Center, $\mathrm{Ti}(\mathrm{i}=1,2)$ is the receiving tower, $\operatorname{Dj}(j=1,2,3)$ is the Line-of-sight among the tower.

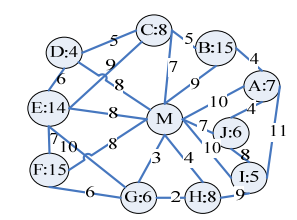

Fig.2 Model diagram

There are three information transmission paths:
Broadcast transmission. That is $\mathrm{C}->\mathrm{T} 1, \mathrm{C}->\mathrm{T} 2, \mathrm{~T} 1->\mathrm{C}, \mathrm{T} 2->\mathrm{C}, \quad$ the total information transmission distance (TITD)

$$
\text { is } \mathrm{A}=2 * \mathrm{D} 1+2 * \mathrm{D} 3 \text {; }
$$

Single path transmission. That is $\mathrm{C}->\mathrm{T} 1->\mathrm{T} 2->\mathrm{T} 1->\mathrm{C}$, the TITD is $\mathrm{B}=2 * \mathrm{D} 1+2 * \mathrm{D} 2$;

Excursion Algorithm. That is $\mathrm{C}->\mathrm{T} 1->\mathrm{T} 2->\mathrm{C}$, the TITD is $\mathrm{C}=\mathrm{D} 1+\mathrm{D} 2+\mathrm{D} 3$.

Now we have to prove: $\mathrm{C}<\mathrm{A}$ and $\mathrm{C}<\mathrm{B}$.

Since $A-C=2 * D 1+2 * D 3-(D 1+D 2+D 3)$, the result is $\mathrm{D} 1+\mathrm{D} 3-\mathrm{D} 2$. According to the Triangle

Theorem, the sum of any two sides of a triangle is great er than the third side, we can get that $\mathrm{D} 1+\mathrm{D} 3-\mathrm{D} 2>0$, that is $\mathrm{A}>\mathrm{C}$, hence, $\mathrm{C}<\mathrm{A}$.

Since $\mathrm{B}-\mathrm{C}=2 * \mathrm{D} 1+2 * \mathrm{D} 2-(\mathrm{D} 1+\mathrm{D} 2+\mathrm{D} 3)$, the result is $\mathrm{D} 1+\mathrm{D} 2-\mathrm{D} 3$. According to the Triangle Theorem, D1+D2-D3 $>0$, that is $\mathrm{B}>\mathrm{C}$, hence, $\mathrm{C}<\mathrm{B}$.

From the result mentioned above, we can get the point that the the Core idea of Excursion Algorithm is correct and achievable. More detailed analysis of the Algorithm will be explained in the following part. We take the value of $\mathrm{A}-\mathrm{C}$ or $\mathrm{B}-\mathrm{C}$ as the saved Transmission value, and $\mathrm{TV}(\mathrm{T} 1, \mathrm{~T} 2)$ represents saved Transmission value from C(Traffic Management Center) passing through $\mathrm{T} 1, \mathrm{~T} 2$ and back to $\mathrm{C}$.

\section{The Process of Excursion Algorithm}

Taking Fig.3 (network diagram) as an example, $M$ is the Traffic Management 
Center, from A-J are 10 receiving towers(10regions), and the unit is MB for the collecting information content in each region. And information content is the nodal value in the fig while the bandwidth is $40 \mathrm{MB}$. In order to avoid too much noise, the transmitting length is below 30 kilometers ${ }^{[7]}$. The edge weight is the line-of-sight (kilometer); the lines connect the receiving towers.

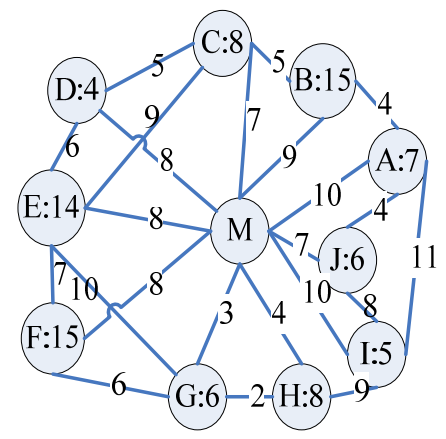

Fig.3 network diagram

\begin{tabular}{|c|c|c|c|c|c|c|}
\hline & M & A & \multirow[t]{3}{*}{$\mathrm{TV}(\mathrm{A}, \mathrm{B})=10+9-4=15$} & & M & A \\
\hline A & 10 & - & & A & 10 & - \\
\hline $\mathrm{B}$ & 9 & 4 & & B & 9 & 15 \\
\hline
\end{tabular}

Fig. 5 the matrix transition diagram

\subsection{The TV Matrix}

From Line A, B (Fig. 5 Left), M->A, and the distance is $10 ; \mathrm{M}->\mathrm{B}$, the distance is 9 ; $\mathrm{A}->\mathrm{B}$, the distance is 4 . Based on the Core idea of Excursion Algorithm, TV (A, B) $=15$ (Fig. 5 Right). Fig. 5 is the matrix
4.1 Figure out the Shortest Path Matrix

Since in wireless transmission, speed is inversely proportional to distance ${ }^{[8]}$, we need to find out the shortest path among receiving towers. Thus, Floyd Shortest Path Algorithm is adapted to put into use here to figure out the shortest path from $M$ to other points as well as the shortest distance among the points $^{[9]}$. Then, we will get the shortest path matrix ${ }^{[10]}$. Shown as in Fig. 4

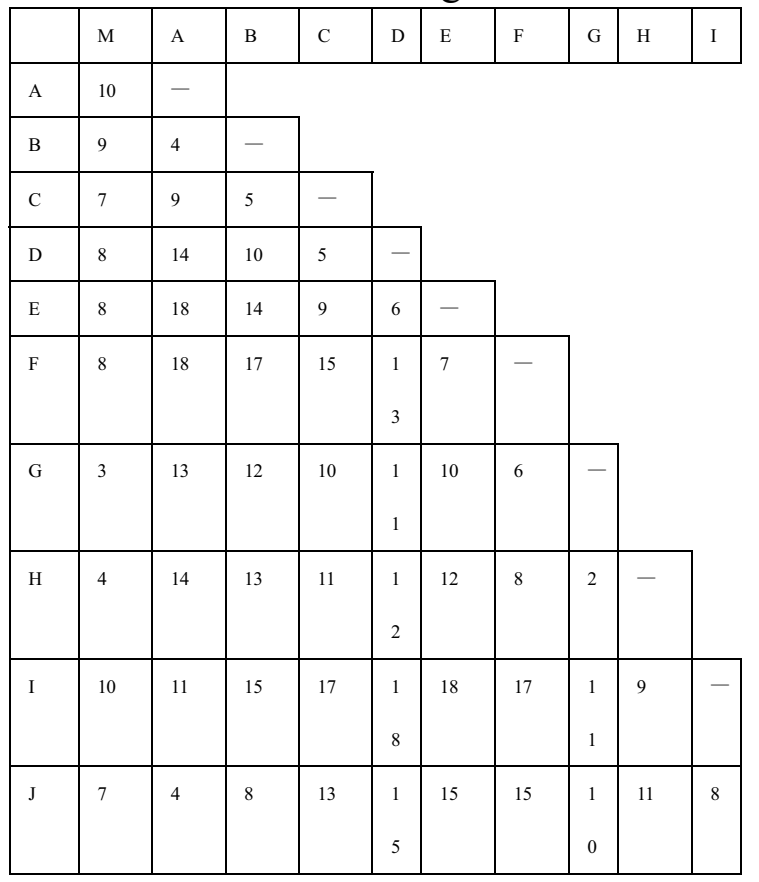

Fig.4 the shortest matrix for each point

transformation diagram of the first two lines.

By examining it, TV $(i, j)=D(i, 0)+$

D (j-1, 0)-D (i, j) can be used to calculate other TVs except M queue. In the matrix, $i$ stand for the line, $\mathrm{j}$ stands for the queue. Thus, $\mathrm{M}$ is queue $0, \mathrm{~A}$ is line $0, \mathrm{TV}(\mathrm{i}, \mathrm{j})$ is the value of Line $i$ and Queue j. In this way, we can get the Fig. 5. 


\begin{tabular}{|c|c|c|c|c|c|c|c|c|c|c|}
\hline & $\mathrm{M}$ & A & B & $\mathrm{C}$ & $\mathrm{D}$ & $\mathrm{E}$ & $\mathrm{F}$ & $\mathrm{G}$ & $\mathrm{H}$ & I \\
\hline$A$ & 10 & - & & & & & & & & \\
\hline B & 9 & 15 & - & & & & & & & \\
\hline $\mathrm{C}$ & 7 & 8 & 11 & - & & & & & & \\
\hline $\mathrm{D}$ & 8 & 4 & 7 & 10 & - & & & & & \\
\hline $\mathrm{E}$ & 8 & 0 & 3 & 6 & 10 & - & & & & \\
\hline $\mathrm{F}$ & 8 & 0 & 0 & 0 & 3 & 9 & - & & & \\
\hline $\mathrm{G}$ & 3 & 0 & 0 & 0 & 0 & 1 & 5 & - & & \\
\hline $\mathrm{H}$ & 4 & 0 & 0 & 0 & 0 & 0 & 4 & 5 & - & \\
\hline $\mathrm{I}$ & 10 & 9 & 4 & 0 & 0 & 0 & 1 & 2 & 5 & - \\
\hline $\mathrm{J}$ & 7 & 13 & 8 & 1 & 0 & 0 & 0 & 0 & 0 & 9 \\
\hline
\end{tabular}

Fig.6 the TV matrix

\begin{tabular}{|l|l|}
$\mathrm{A}-\mathrm{B}$ & 15 \\
\hline $\mathrm{A}-\mathrm{J}$ & 13 \\
\hline $\mathrm{B}-\mathrm{C}$ & 11 \\
\hline $\mathrm{C}-\mathrm{D}$ & 10 \\
\hline $\mathrm{D}-\mathrm{E}$ & 10 \\
\hline $\mathrm{A}-\mathrm{I}$ & 9 \\
\hline $\mathrm{E}-\mathrm{F}$ & 9 \\
\hline $\mathrm{I}-\mathrm{J}$ & 9 \\
\hline $\mathrm{A}-\mathrm{C}$ & 8 \\
\hline $\mathrm{B}-\mathrm{J}$ & 8 \\
\hline $\mathrm{B}-\mathrm{D}$ & 7 \\
\hline $\mathrm{C}-\mathrm{E}$ & 6 \\
\hline $\mathrm{F}-\mathrm{G}$ & 5 \\
\hline $\mathrm{G}-\mathrm{H}$ & 5 \\
\hline $\mathrm{H}-\mathrm{I}$ & 5 \\
\hline$\cdots$ & $\ldots$ \\
\hline$\cdots$ & $\cdots$ \\
\hline
\end{tabular}

Fig.7 the TV queue

From Fig. 6, the value represents the saved transmission value of the paths. Taking A-J as an example, the transmission distance can be saved up to 13 kilometers. However, with the constraints of $40 \mathrm{M}$ bandwidth and under 30 kilometers transmitting distance, not all the paths are the selection paths.

\subsection{Path Selection Process.}

The higher TV, the shorter distance. So we sort the information in Fig. 6 in descending order (Fig. 7) to get the TV queue. Dequeening it one by one, we will get the Conditional
Additive Queue. As queueing, the value of the distance and the amount of the information will be added. Once the meeting the constraints of $40 \mathrm{M}$ bandwidth and 30 kilometers transmitting distance, the value ranged in the last place will be dequeened with the path deleted. At the same time, the queueing path will be marked on the network diagram (deleting all the paths in Fig. 3 except the direct transmission from $\mathrm{M}$ ). The marked path will form a Excursion Algorithm. The Path selection process is shown in Fig. 8. 


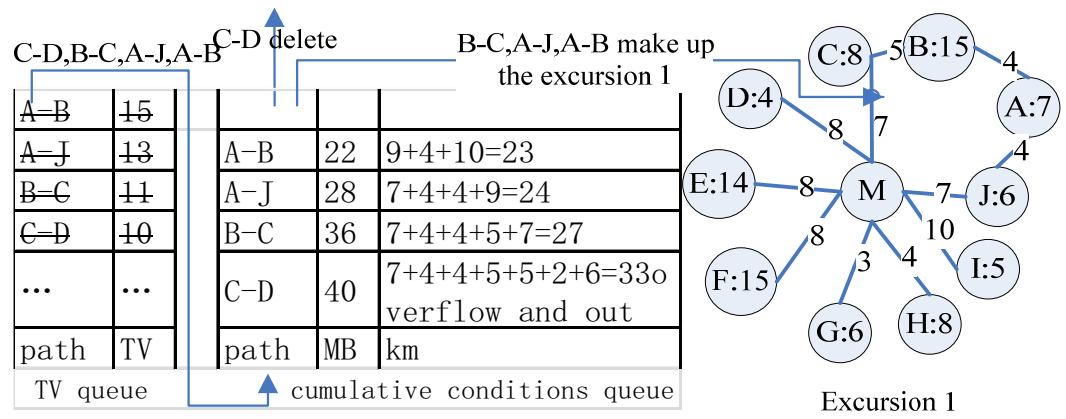

Fig. 8 the process diagram about the path choice

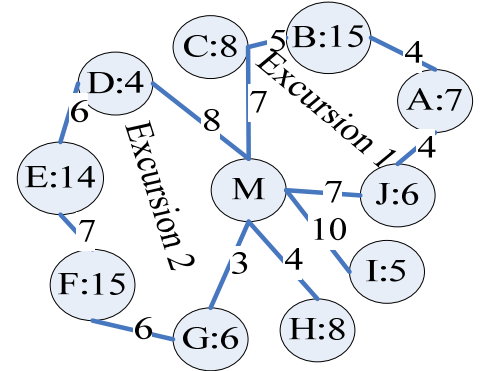

Fig. 9 the diagram about the second Excursion

Similar processes as the first Excursion, from $\mathrm{D}-\mathrm{E}$ to $\mathrm{G}-\mathrm{H}$, the value are dequeened. Thus, A-I,I-J,A-C,B-J,B-D,C-E cannot be added back to the Excursion- value will not be marked on the network diagram. The process of D-E, E-F, F-G, G-H is the same as

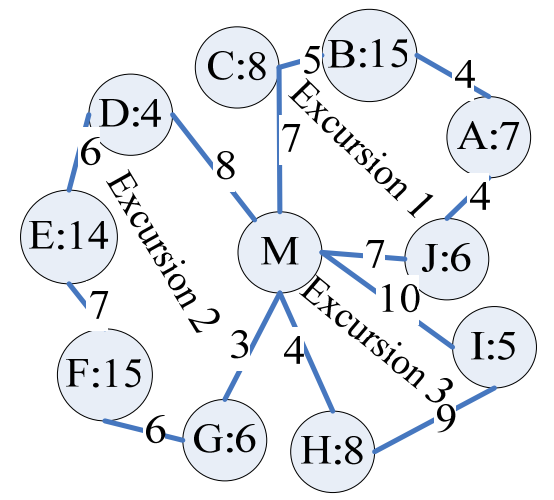

Fig.10 The final Routing Scheme use of Excursion

\section{Performance Analysis}

Through OPNET, we make a comparison on the Cellular System, simple Broadcast the one in Fig. 8. When G-H is adding to the Conditional Additive Queue, the distance is over 30 kilometers; it will form the second Excursion (Fig. 9). By repeating the procedure above, we will get the final Excursion Algorithm (Fig. 10).

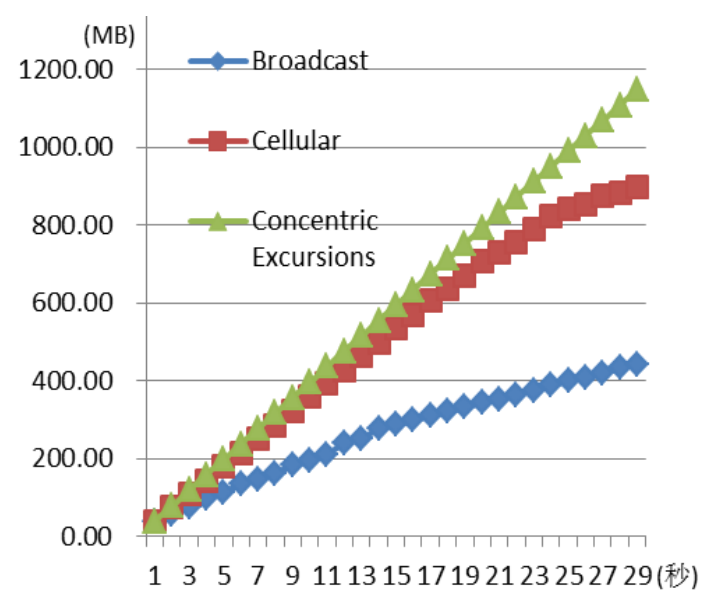

Fig.11 The throughput comparison chart

Method and Routing Excursion Algorithm. In the case of the same environment and parameters (the Node number and location in Fig.3), the throughput capacity is shown in 
Fig.3 (The throughput comparison chart). From the chart, the basic linear of Routing Excursion Algorithm grows with the time, while the Cellular System tends to be lagging behind with the growth of time. And the Broadcast Method is quite slow in growing.

\section{Conclusion}

Routing Excursion Algorithm here is using the Traffic Management Center as the center to send out information to all the other regions as well receives the signals. It has to meet the demand of working under limiting condition as well to keep high efficiency. Besides routing method, there are still plenty factors may influence the efficiency of the net, such as the transmitting power of wireless devices, the relay capability of the receiving towers, the computational efficiency of the server and etc.. In this article, the author did not take them into consideration. However, these factors can be treated as numerical formation condition which can also be solved out with the method of this article. Sincerely hope that the readers can get help after reading this article.

\section{Acknowledgments}

Funds supported by 2014 Guangdong Province Outstanding Young Teachers Training program Funded Project (Grant No. 2015S02); 2013 Guangdong Province Outstanding Young Teachers Training program Funded Project (Grant No: Yq2013201); High Tech Industrialization Project of Guangdong Science and Technology Department of (Grant No: 2014A010103002); Special Funds for Demonstration Projects of Dongguan Polytechnic College (Grant No: ZXHQ2014d001).

\section{References}

[1] ZHAO Na, YUAN Jia-bin, XU Han. Survey on Intelligent Transportation System [J]. Computer Science, 2014,41(11): 7-11.

[2] Lu Huapu, Li Ruimin. Developing Trend of ITS and Strategy Suggestions[J]. JOURNAL OF ENGINEERING STUDIES, 2014, 06(1): 6-19.

[3] Wang Shundao, Song Rongfang. Joint optimization of sensing time and power allocation in small-cell networks [J]. Application Research of Computers, 2015,32(9),2766-2768.

[4] ZHOU Xian-wei, LIU Bin, QIN Bo-ping. Research on Routing Algorithm for Wireless Sensor Network [J]. Journal of Transduction Technology, 2006,19(2): 463-467.

[5] ZHANG Jian-kui,QIAN Meng-tao,QIAN Chuan-gen. Analysis and optimization of wireless network structure on the miniature type and medium-sized city [J]. Jiangsu Communication. 2011,(3): 58-60.

[6] JI X Y. Models and algorithm for stochastic shortest path problem. Applied Mathematics and Computation . 2005

[7] Luo Jianfeng Ji Shanshan Zheng Huijun. Design of Algorithm for Network Information Search in Information Integrated Platform of Cargo Tracking[J]. Science Mosaic, 2015,(2):51-53.

[8] LUO Kun WANG Jian-xin ZHAO Xiang-ning. Survey on Geographic Routing in Wireless Sensor Networks [J]. Computer Science, 2008,35(10): 28-32.

[9] WEI D C. An optimized Floyd Algorithm for the shortest path problem. Journal of Networks . 2010

[10] Da-chuan Wei. Implementation of route selection function based on improved Floyd algorithm. Proceedings of the 2010 WASE international conference on information engineering . 2010 